\title{
How Do the Users Show their Interest on Line?----- Eye Movement and Browsing Behaviour
}

\author{
Xiaowei Shi ${ }^{1}$, Zhi Gu ${ }^{2}$, Daofang Chang ${ }^{3}$ and Linping Huang ${ }^{4}$ \\ ${ }^{1}$ Department of Logistics Engineering, Shanghai Maritime University, Shanghai, \\ China \\ ${ }^{2}$ Logistics Research Center, Shanghai Maritime University, Shanghai, China \\ ${ }^{3}$ Department of Logistics Engineering, Shanghai Maritime University, Shanghai, \\ China \\ ${ }^{4}$ Department of Technology, Shanghai Yuhong Information \& Technology Co, \\ Ltd., Shanghai, China \\ guzhiczu@163.com
}

\begin{abstract}
Nowadays, increasing number of people prefer to shop online which has become a central part in their daily life. However, this precipitates a need to get knowledge about how people show their interests in the product which may contribute to recommend the right product for them. In this paper, an experiment was conducted in a clothing website which aimed to find out how and when people were interested in certain product in terms of eye movement and browsing behaviour. The results show that for eye movement, there is no direct relationship between pupil diameter and interests, and user's interests have direct correlativity with blink frequency for some users. Moreover, eye movement can predict the user's interests, and the accuracy is about 62\%; for browsing behaviour, scroll times and display time also has something to do with the user's interest; as for the relationship between eye movement and interactive behaviour, when scroll times increases, then saccade will increases too, but fixation time will reduce. Therefore, the conclusion can be drawn that when users shop online, they always need to interact with online store, and eye movement and interactive behaviour occurs in a particular pattern. This result could contribute to choose the corresponding parameters for recommendation and avoid using the parameters which can reflect users' interests in the same degree or choose the one which can reflect users' interest effectively to greatest extent.
\end{abstract}

Keywords: eye movement; interactive behaviour; interaction; on line shopping

\section{Introduction}

Nowadays the Internet has become more and more popular and important in both daily life and business. In particular, most of people prefer to shop online such as eBay, Amazon, etc., which just as popular as people get accustomed to go shopping in the shopping mall. As for some people who are addicted to shop online, they would even like to visit shopping website just for fun or waiting for something cheap to buy. Therefore, there is no doubt that online shopping has played a key role in consumption patterns of modern people.

In fact, typical Internet users are overwhelmed by the ever-increasing amount of commodities available on the World Wide Web, waiting to be consumed by millions of people connected to the Internet. However, the large amount of commodities not only brings the various kinds of commodities information, but also contributes to information overload which leads to the difficulties for finding the commodities that interests users. 
As a result, people have to take enormous time to find goods that is satisfying, which sometimes may make them feel frustrated.

According to this tricky problem, a varied inventory of researchers try to solve it in terms of recommendation algorithms, users' interactive behaviours with shopping website, shopping website layout and so forth. Even though it is of great importance to research on recommendation algorithms, website layout, etc., to understand users' interaction behaviours is a foundation of this theories. This precipitates a need to get knowledge about how people show their interests in the products which may, in great extent, contribute to recommend the appropriate product for them on the internet.

According to the research, users' need include explicit needs and implicit needs [1]. In fact, users' interests are changing over time, whereas users' preferences are changing according the viewer's gradual maturity. It is incomplete to acquire user preference only depending on user's effort. What is more, some users may feel antipathy to express their explicit needs because they have to fill forms or type something into computer or maybe just do not know what they want. Just depending on filling out a form when they registered may even lead to misunderstand users' real interests because of the over time changing. Thus, it necessitates researchers to pay attention to users' implicit needs.

In this paper, interests refer to something that concerns, involves, draws the attention of or arouses the curiosity of a person. When users are interested in something, there is some change may happen both in physical and psychological state. While users' psychological state is fairly difficult to be observed directly, physical state can be observed and recorded easily, such as eye movement. Other than eye movement, user's interactive behaviours with shopping website also more or less reflect users' preference. In order to solve this problem, researchers focus on two metrics that could measure interest of users effectively, that is to say, interactive behaviour and eye movement.

Therefore, this paper aims to explore users' implicit interests to resolve the problem that is hard for users to find what they are interested in with such large amount of products. In order to achieve this goal, both of interactive behaviours, eye movement of online shopping buyers and corresponding interest degree of goods are recorded simultaneously by conducting an experiment with Smart eye system and a website which can record users' interactive behaviours. By observing these data, the relationship between users' interest and their interactive behaviour, users' interest and eye movement are analyzed. Interactive behavioural involves scroll, display time; eye movement includes fixation, saccade, blink, pupil diameter, and eyelid opening. In the other hand, the relationship between Interactive behaviour and eye movement are analyzed as well in consideration of some parameters having the same function relating to the interests.

\section{Related Works}

In recent decades, the network technique has been advanced by leaps and bounds, and surfing on the internet becomes increasingly popular. Thus, to understand users' interest on the net becomes much more important now than in the past. Therefore, much interest has concentred around the fact that it is also possible to identify users' interest.

Interest refers to a kind of special emotion that can draw people's attention to, like or care about something. For psychologist, interest is a kind of conscious tendency to know something or participate in some activities positively. In fact, interest could be shown by people's actions. That is to say, people could understand the degree people are interested in something by people's actions. For example, Jiahui Liu, etc develop a personalized news recommendation based on users' past click behaviour to find the news that users may be interested in [2]. Matthew Michelson, etc. research on discovering users' topics of interest on Twitter by what they mention about [3].

In the other hand, in order to get higher precision of recommendation, there are a large amount of people tried to understand which behaviour could represent people's interest 
best. According to actions from different part of body, parameters that could reflect user's interest can be distinguished into interactive behaviour and eye movement.

Interactive behaviour refers to the behaviour users interact with browser, or webpage. The research on user's interests which is based on the interaction data that is easier to get than eye movement, such as click through, scroll, display time, mouse moving [4-8], etc.

Some literatures mention that eye movement may relate to user's intention [9-11] or emotion [12]. In fact, 20 centuries ago, people have already researched on eye movement analysis. Psychologists explored the direct or indirect relationship between eye movement and people's psychological activity in different context. In addition, eye movements have been turned out to be very efficient parameters to provide a useful implicit feedback channel in reading area [13].

Hence, it is worthy to explore the relationship between user's interests and interaction behaviour, eye movement. This paper aims to explore whether or not there exist relationships between users' interests and users' eye movements, historical behaviour in e-commerce field. The exploration will be conducive to predict user's preference accurately in the future study and help people develop more precise recommendation system.

\subsection{Materials}

The parameters of eye movement are collected by Smart Eye System within Microsoft Windows XP and Smart Eye System is made in Switzerland. The Smart Eye Pro System involves a monitor (version is L2250pwD), a Lenovo host (Intel (R), Corel (TM) 2 Duo, 1.98 GB of RAM), and an Exponator 5 and four cameras (Sony HR-50 $8.0 \mathrm{~mm}$ lens) with iris flashes. Smart Eye Pro 5.6 system is installed on the computer, and the system is used to record the eye movements of participants. For fear of annoying or bothering the participants and influencing on the validity of experimental data, another monitor (version is L2250pwD) is added, one monitor is shown for investigators and another is shown for the participants. Two monitors are connected on the same host, and show the same contents. Figure 1 shows the how the hardware is installed.

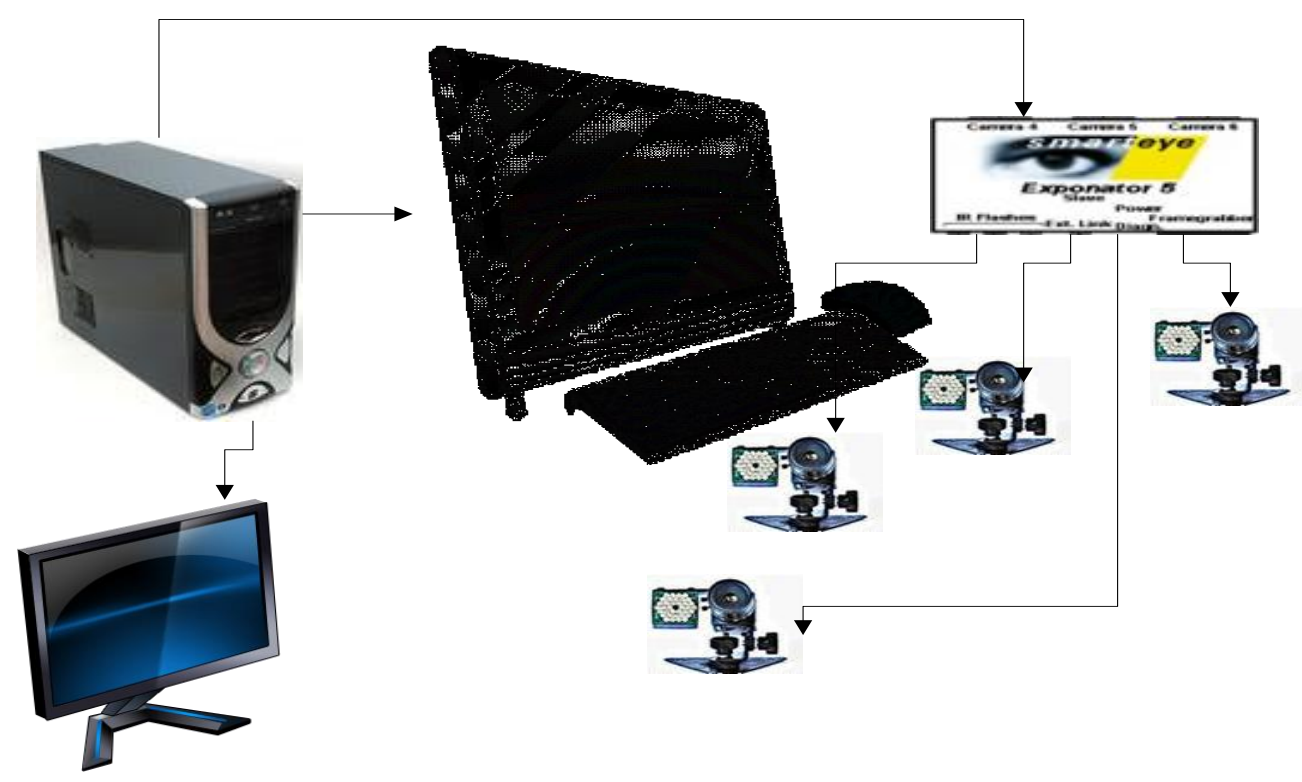

Figure 1. Hardware in the Experiment 


\subsection{Website}

The fashion website as in Figure 2 studied in this experiment is constructed by one of experimenters, which aims to record the interactive behaviour of participants in the database for data analysis. The clothes information is collected from the website of www.taobao.com, a most popular e-commerce website in China. 6 hundred of product information is collected and they are classified according to style, price, gender, quality, and popular level, and so on. The data is easy to be acquired and people buy these clothes pretty frequently, in addition, the description for the product is attractive and complicated enough, involving brand, price, style, etc.

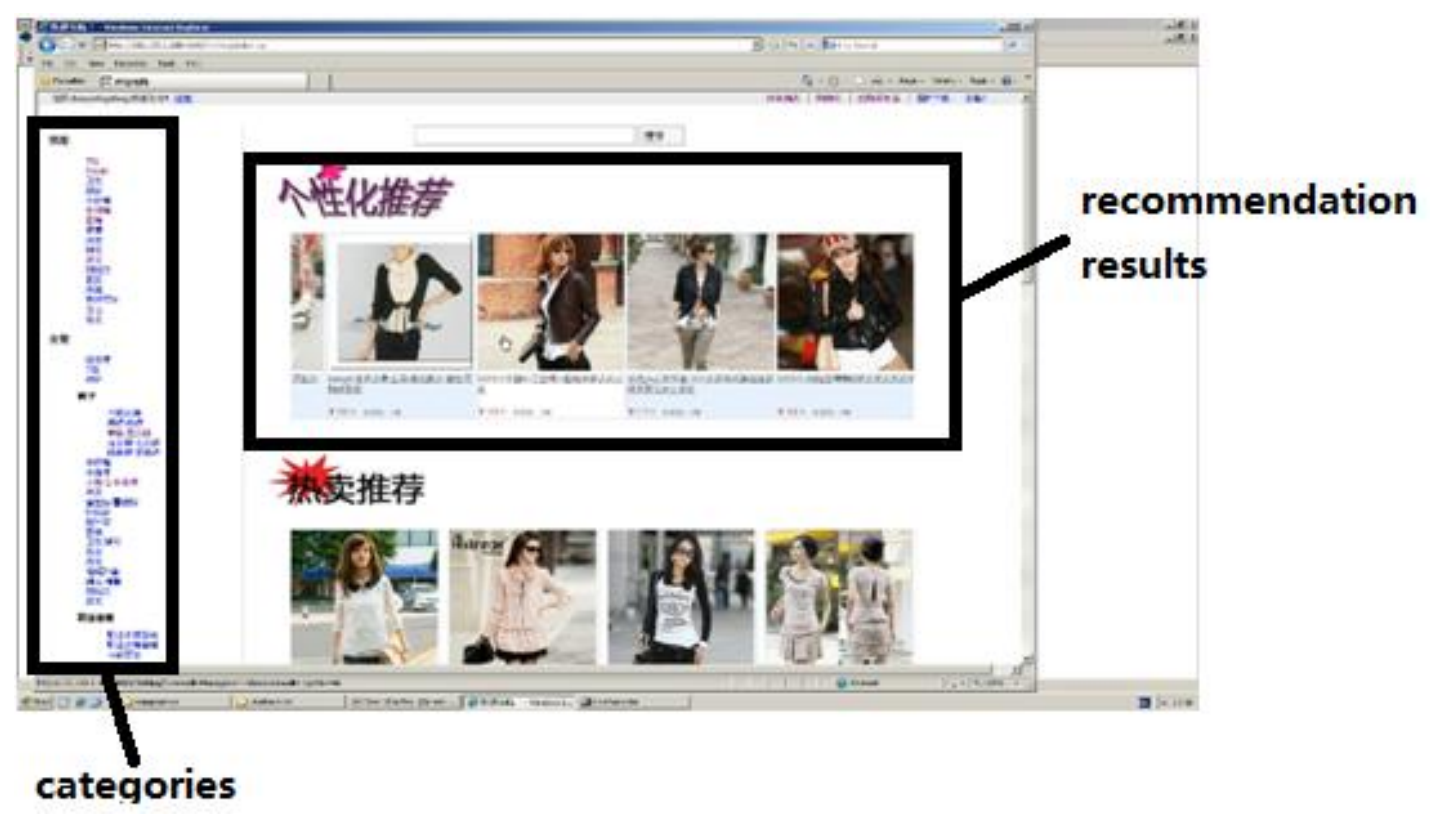

Figure 2. Fashion Website

In this website, users can search clothes on the search bar, or according to the classification, or even find them on the recommendation column. The interactive behaviour of users can be logged in the database, and the behaviours include buy clothes, open the product descriptive pages, close the product descriptive pages, and scroll the webpage, and so on.

\subsection{Participant}

There are 40 participants in this experiment. Their age is range from 16 to 43 , and all of them are familiar with Windows System. Their average time they spent on computer per day is about 6.5 hours and their average vision acuity is range from 4.5 to 5.0.

\subsection{Procedure}

Step 1: Introduction for participants involved experiment procedure and participants should rank the clothes they browsed as in Table 1.

Table 1. Interested Degree for Clothes

\begin{tabular}{|c|c|c|c|c|c|}
\hline & very interested & interested & moderate & hate & very hate \\
\hline $\begin{array}{c}\text { interest } \\
\text { degree }\end{array}$ & 5 & 4 & 3 & 2 & 1 \\
\hline
\end{tabular}


Step 2: Participants logged in the website and registered on the website;

Step 3: Participants's eye models were constructed in Smarteye system and their eye tracking precision should be adjusted as well;

Step 4: Participants logged in the website again, and browsed the clothes for about 10 minutes without any intervene. If collapsed, experimenter would restart it with the constructed eye model.

\section{Result}

In this study, the number of fixation point is collected about 1644 every participant, and the total number of fixation point collected is 65760 . Participants evaluate the like degree of each product webpage they visit. The webpage is included: search page and product descriptive page. The eye movement on this page is tracked involves fixation, saccade, blink; the browsing behaviour includes scroll, display time, etc.

\subsection{Eye Movement Analysis}

Eye movement parameters which are analyzed here include fixation time, saccade time, blink time, pupil diameter and eyelid opening. However, for the convenience of analysis, data is processed as follows:

$$
\begin{gathered}
\mathrm{F}_{\text {frequency }}=T_{\text {fixation time }} /\left(\mathrm{T}_{\text {fixation time }}+T_{\text {saccade time }}+T_{\text {blink time }}\right) \\
S_{\text {saccade }}=T_{\text {saccade time }} /\left(\mathrm{T}_{\text {fixation time }}+T_{\text {saccade time }}+T_{\text {blink time }}\right) \\
B_{\text {blink }}=T_{\text {blink time }} /\left(\mathrm{T}_{\text {fixation time }}+T_{\text {saccade time }}+T_{\text {blink time }}\right)
\end{gathered}
$$

Where $\mathrm{F}_{\text {frequency }}$ indicates fixation frequency; $S_{\text {saccade }}$ indicates saccade frequency, $B_{\text {blink }}$ indicates blink frequency; $\mathrm{T}_{\text {fixation time }}$ means fixation time; $T_{\text {saccade time }}$ refers to saccade time; $T_{\text {blink time }}$ indicates blink time.

$$
A_{\text {pupil diameter }}=\left(\sum_{\text {in product webpage }} P_{\text {pupil diameter }}\right) / T_{\text {frame }}
$$

Where $A_{\text {pupil diameter }}$ indicates the average value of pupil diameter; $P_{\text {pupil diameter }}$ means pupil diameter every frame in certain product webpage; $T_{\text {frame }}$ refers to the total number of frame in certain product webpage.

$$
A_{\text {eyelid opening }}=\left(\sum_{\text {in product webpage }} E_{\text {eyelid opening }}\right) / T_{\text {frame }}
$$

Where $A_{\text {eyelid opening }}$ indicates the average value of pupil diameter; $E_{\text {eyelid opening }}$ means eyelid opening every frame in certain product webpage; $T_{\text {display time }}$ refers to the total number of frame in certain product webpage.

These parameters and interested degree that participants evaluate are applied Pearson Correlation Analysis. The result shows that there is a negative correlation between blink frequency and fixation frequency $(\mathrm{r}=-0.508, \mathrm{p}=0.008)$ and there is also a negative correlation between saccade frequency and fixation frequency $(\mathrm{r}=-0.912, \mathrm{p}=0.000)$. For some experiment, there is direct correlation between blink frequency and people's interest degree.

In some literatures, they mention that pupil diameter can predict how much people are interested. However, the result shows that there is no direct relationship between pupil diameter and people's interest in this experiment, but there is only positive correlation between pupil diameter and eyelid opening. 
An interesting phenomenon is found when data is processed as follows:

$$
\text { Fixation }_{\text {this page }}=\mathrm{F}_{\text {total frequency }}-\mathrm{F}_{\text {frequency in this page }}
$$

Where Fixation $_{\text {this page }}$ indicates how much the fixation frequency are increased or

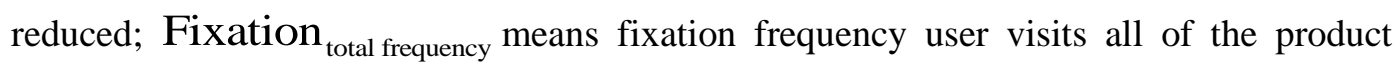
descriptive webpage; $F_{\text {frequency in this page }}$ refers to fixation frequency that user browses certain product descriptive webpage.

$$
\text { Saccade }_{\text {this page }}=\mathrm{S}_{\text {otal frequency }}-\mathrm{S}_{\text {irequency in this page }}
$$

Where Saccade $_{\text {this page }}$ indicates how much the saccade frequency are increased or reduced; $S_{\text {total frequency }}$ means saccade frequency user visits all of the product descriptive webpage; $S_{\text {frequency in this page }}$ refers to saccade frequency that user browses certain product descriptive webpage.

$$
\text { Blink } k_{\text {this page }}=B_{\text {total frequency }}-B_{\text {frequency in this page }}
$$

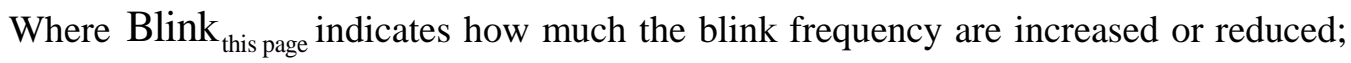
$\mathrm{B}_{\text {total frequency }}$ means blink frequency user visits all of the product descriptive webpage; $B_{\text {frequency in this page }}$ refers to blink frequency that user browses certain product descriptive webpage.

$$
E_{\text {this page }}=E_{\text {total frequency }}-E_{\text {frequency in this page }}
$$

Where $\mathrm{E}_{\text {this page }}$ indicates how much the eyelid opening are increased or reduced; $\mathrm{E}_{\text {total frequency }}$ means eyelid opening user visits all of the product descriptive webpage; $E_{\text {frequency in this page }}$ refers to eyelid opening that user browses certain product descriptive webpage.

$$
P_{\text {this page }}=P_{\text {total }} \quad \text { frequency } P \text { frequency inthige }
$$

Where $\mathrm{P}_{\text {this page }}$ indicates how much the pupil diameter are increased or reduced; $\mathrm{P}_{\text {total frequency }}$ means pupil diameter user visits all of the product descriptive webpage; $P_{\text {frequency in this page }}$ refers to pupil diameter that user browses certain product descriptive webpage.

Suppose that three of the four parameters, that is, Fixation this page, Saccade this page, Blink $_{\text {this page, }} \mathrm{E}_{\text {this page, }}, \mathrm{P}_{\text {this page, }}$, is larger than 0 , which indicates participants like this product, then the accuracy to predict that user like the product is $62 \%$. The result shows that it is useful to predict whether user is interested in the products.

\section{2 . Browsing Behaviour Analysis}

It can be seen from Figure 3 that when people visit the website, the behaviour involves left mouse button pressed/ released, mouse wheel, window opened and window maximized happens fairly frequently. 


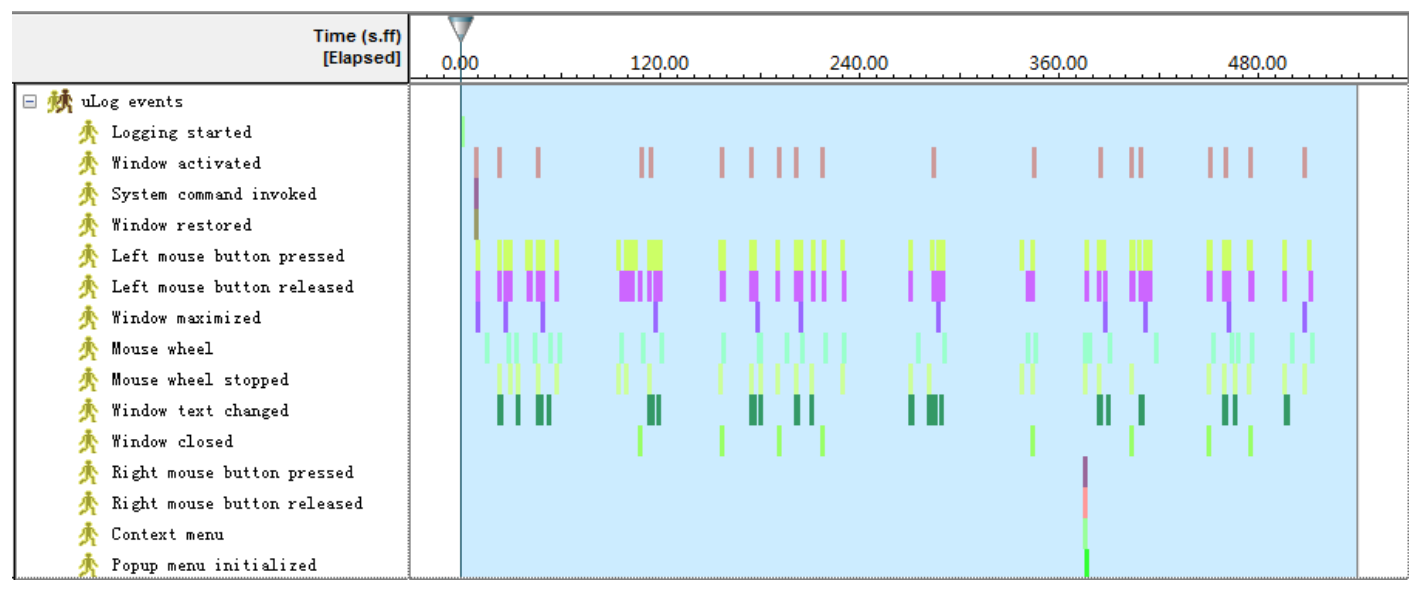

Figure 3. Gantt Chart about How to Behave When People Browse the Product Descriptive Webpage

However, this section only study on the browsing behaviour visiting the production descriptive webpage, so click, open and close webpage happens rarely. For this reason, click will not be studied.

(1) Scroll

In this section, Pearson Correlation Analysis is applied between scroll frequency and interested degree that participants evaluate, and between scroll times and interested degree. The result shows that there exists the low correlation between user's interest and scroll frequency $(\mathrm{r}=0.405, \mathrm{p}=0.045)$, which indicates scroll frequency can, in some extent, reflect user's interest.

(2) Display time

The result of Pearson Correlation analysis shows that the display time has a positive correlation with user's interest $(\mathrm{r}=0.822, \mathrm{p}=0.023)$.

It can be seen from Table 2 that the display time people visit the product descriptive webpage that they are interested in is longer than the webpage they are not.

Table 2. The Average Display Time of Interested and Not Interested Product Descriptive Webpage

\begin{tabular}{|c|c|c|}
\hline & $\begin{array}{c}\text { interested product } \\
\text { descriptive webpage }\end{array}$ & $\begin{array}{c}\text { not interested product } \\
\text { descriptive webpage }\end{array}$ \\
\hline average display time & 24.3 & 20.7 \\
\hline
\end{tabular}

\subsection{Interaction between Eye Movements and Browsing Behaviour}

There is a positive correlation between scroll frequency and saccade frequency $(\mathrm{r}=0.584, \mathrm{p}=0.002)$, in contrast, a negative correlation between scroll frequency and fixation frequency $(\mathrm{r}=-0.639, \mathrm{p}=0.000)$. Therefore, the scroll frequency is increased, then saccade frequency is increased too, but fixation frequency is reduced.

When people want to find the products they need, their fixation and saccade pattern will be as in Figure 4. They will repeat the fixation-saccade-fixation pattern, finally, they will fixate on certain place and move the mouse to the click the link of the product they are interested in. 


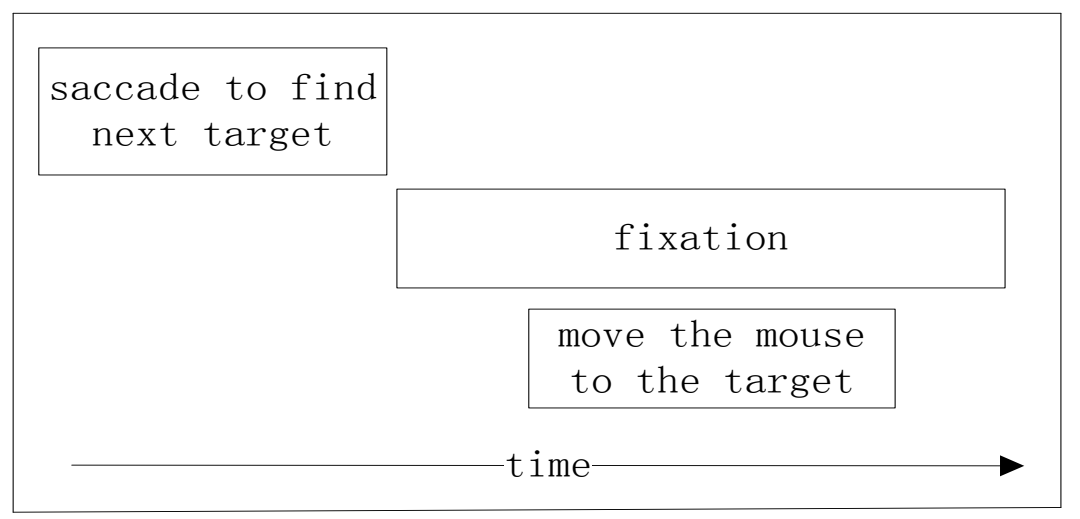

\section{Figure 4. Interaction between Eye Movement and Browsing Behaviour}

When people visit the descriptive webpage and find some information they are interested in, they will slow down or stop scroll, and their eye movement will change from saccade to fixation, as in Figure 5. This means they are processing the information they are interested in.

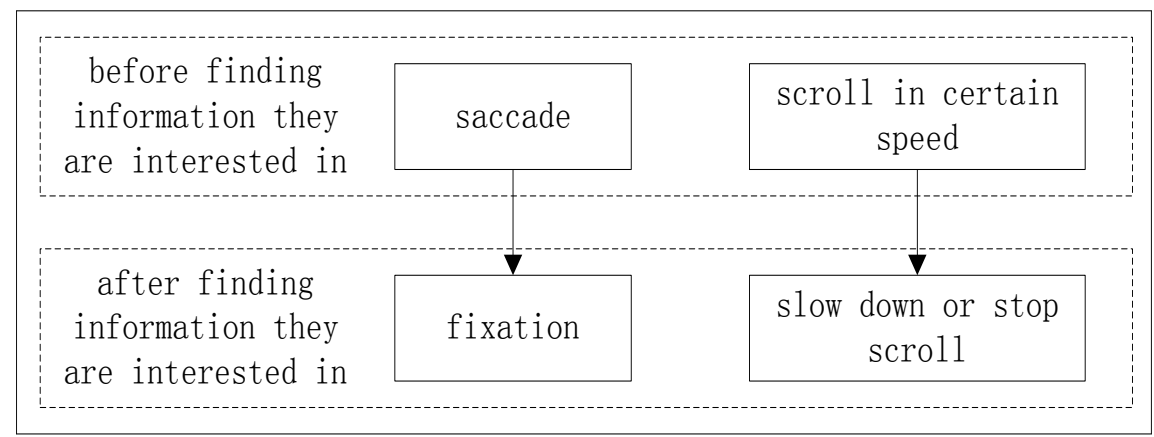

\section{Figure 5. The Eye Movement Behaviour and Browsing Behaviour Before and After People Find Some Information they are Interested in}

\section{Discussion and Conclusion}

As a whole, it can be seen from the result that the scroll frequency has a directly positive correlation with user's interest, and has a positive correlation with saccade frequency, and however, has a negative correlation with fixation. Therefore, a conclusion can be drawn that user's interest can be judged from saccade frequency and fixation frequency. In addition, both blink frequency and display time has a positive correlation with user's interest. Thus, the time user visits product descriptive webpage is longer, then the blink will more frequent.

The layout of the web pages is similar and familiar for user, thus user will only pay attention to the information they are interested in and overlook the information they are not interested in. Moreover, when user finds something they are interested in, they will slow down or stop the scroll behaviour and start to fixate on the information. This is mainly for two reasons: firstly, they need time to process the information; secondly, people always cannot help appreciating what they like.

However, there are still some issues left. In order not to interrupt the experiment and make subjects feel like they are shopping on their will rather than performing an experiment, the precision of eye movement of subjects was not adjusted during the 
experiment and thus kept falling down slowly. We believe these challenges will require more substantial thinking in our experiment for further research.

\section{Acknowledgements}

This work sponsored by National 863 plans projects (2009AA043001), supported by Shanghai Maritime University Research Project (20110019\&20110316).

\section{References}

[1] R. W. White, J. M. Jose and I. Ruthven, "Comparing implicit and explicit feedback techniques for web retrieval: TREC-10 interactive track report", Proceedings of the Text Retrieval Conference (TREC 2001). Gaithersburg, Maryland, USA. (2001).

[2] J. Liu, P. Dolan and E. R. Pedersen, "Personalized News Recommendation based on Behavior", Proceedings of the $15^{\text {th }}$ International Conference on Intelligent User Interfaces, (2010), February 07-10, Hong Kong, China.

[3] M. Michelson and S. A. Macskassy, "Discovering Users' Topics of Interest on Twitter: A First Look", Proceedings of the fourth workshop on Analytics for noisy unstructured text data, (2010), October 26, Toronto, ON, Canada.

[4] S. Fox, K. Karnawat, M. Mydland, S. Dumais and T. White, "Evaluating implicit measures to improve web search", ACM Transactions on Information Systems (TOIS), vol. 23, no. 2, (2005) April, pp. 147168.

[5] T. Joachims, L. Granka, B. Pan, H. Hembrooke, Geri Gay, Accurately interpreting clickthrough data as implicit feedback, Proceedings of the 28th annual international ACM SIGIR conference on Research and development in information retrieval, August 15-19, 2005, Salvador, Brazil.

[6] T. Joachims and F. Radlinski, "Search Engines that Learn from Implicit Feedback", Computer, vol. 40, no. 8, (2007) August, pp. 34-40.

[7] D. Kelly and J. Teevan, "Implicit feedback for inferring user preference: a bibliography", ACM SIGIR Forum, vol. 37, no. 2, Fall (2003), pp. 18-28.

[8] M. Claypool, P. Le, M. Wased and D. Brown, "Implicit interest indicators", Proceedings of the 6th international conference on Intelligent user interfaces, pp. 33-40, January 14-17, 2001, Santa Fe, New Mexico, United States.

[9] G. Buscher, A. Dengel and L. van Elst, "Eye movements as implicit relevance feedback", CHI '08 extended abstracts on Human factors in computing systems, (2008) April 05-10, Florence, Italy.

[10] G. Buscher, A. Dengel and L. V. Elst, "Query expansion using gaze-based feedback on the subdocument level", Proceedings of the 31st annual international ACM SIGIR conference on Research and development in information retrieval, (2008), July 20-24, Singapore, Singapore.

[11] J. Salojarvi, K. Puolamäki, and S. Kaski, "Implicit relevance feedback from eye movements", In ICANN'05, (2005), pp. 513--518.

[12] I. Arapakis, J. M. Jose and P. D. Gray, "Affective feedback: an investigation into the role of emotions in the information seeking process", Proceedings of the 31st annual international ACM SIGIR conference on Research and development in information retrieval, (2008) July 20-24, Singapore, Singapore.

[13] A. Ajanki, D. Hardoon, S. Kaski, K. Puolamäki and J. Shawe-Taylor, "Can Eyes Reveal Interests? Implicit Queries from Gaze Patterns”, User Model User-Adap Inter, vol. 19, no. 4, (2009), pp. 304- 339.

\section{Author}

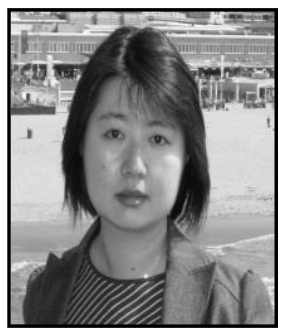

Xiaowei Shi, female, she was born in 1973, doctor degree. Major research field: Human-computer interaction, ergonomics application. 
International Journal of Multimedia and Ubiquitous Engineering Vol.10, No.4 (2015) 